Article

\title{
Stochastic Thermodynamics of Brownian Motion
}

\author{
Grégoire Nicolis $^{1, *}$ and Yannick De Decker ${ }^{1,2}$ \\ 1 Center for Nonlinear Phenomena and Complex Systems (CENOLI), Université Libre de Bruxelles (ULB), \\ Campus Plaine, C.P. 231, B-1050 Brussels, Belgium; ydedecke@ulb.ac.be \\ 2 Nonlinear Physical Chemistry Unit, Université Libre de Bruxelles (ULB), Campus Plaine, C.P. 231, \\ B-1050 Brussels, Belgium \\ * Correspondence: gnicolis@ulb.ac.be
}

Received: 29 June 2017; Accepted: 14 August 2017; Published: 23 August 2017

\begin{abstract}
A stochastic thermodynamics of Brownian motion is set up in which state functions are expressed in terms of state variables through the same relations as in classical irreversible thermodynamics, with the difference that the state variables are now random fields accounting for the effect of fluctuations. Explicit expressions for the stochastic analog of entropy production and related quantities are derived for a dilute solution of Brownian particles in a fluid of light particles. Their statistical properties are analyzed and, in the light of the insights afforded, the thermodynamics of a single Brownian particle is revisited and the status of the second law of thermodynamics is discussed.
\end{abstract}

Keywords: stochastic thermodynamics; brownian motion; nonequilibrium thermodynamics; irreversibility

\section{Introduction}

Classical irreversible thermodynamics provides a unifying description of macroscopic systems out of equilibrium in terms of a limited number of variables satisfying balance equations, supplemented by appropriate phenomenological relations linking the fluxes of the various processes present to the associated thermodynamic forces [1-3]. These equations lead in turn to a balanced equation for entropy from which the entropy production, measuring irreversibility at the thermodynamic level of description, can be identified. On the other hand, increasing recent attention on nanoscale systems or on systems of restricted geometry as they arise, for instance, in cellular and molecular biology and in materials science has highlighted the need to address the role of microscopic-level processes on the evolution of the macroscopic observables. This has led to the development of stochastic thermodynamics [4-11], whose objective is to reassess the laws of classical thermodynamics, by incorporating information pertaining to the fluctuations, the spontaneous deviations from the values provided by the phenomenological laws of evolution arising from microscopic-level processes. Of special interest in this context are the new insights on the status of the second law of thermodynamics afforded by extending the concept of entropy production at the nanoscale.

Recently, the present authors developed an approach to stochastic thermodynamics based on an extended local equilibrium Ansatz [12,13]. The main idea is that state functions such as entropy are expressed in terms of the state variables through the same relations as in classical irreversible thermodynamics, with the difference that the state variables are now random variables whose evolution accounts for the effect of the fluctuations. In the present work this approach is used to set up a stochastic thermodynamics of Brownian motion.

Brownian motion is one of the prototypical examples used to illustrate current approaches to stochastic thermodynamics. Considerable effort has been made to identify thermodynamic quantities like work, heat or entropy at the level of a single Brownian particle [6,11,14-17]. On the other hand, 
much less attention has been paid to the stochastic thermodynamics of a collection (a binary solution) of Brownian particles embedded in a fluid $[11,18,19]$. One of our aims is to establish the fluctuating thermodynamics of such a system, which is much closer to the type of systems considered in classical irreversible thermodynamics, and to assess the role of collective effects in the thermodynamic properties. This will allow us to clarify the status of quantities such as entropy production usually introduced ad hoc at the single particle level and bridge the gap between macroscopic non-equilibrium thermodynamics and more microscopic approaches.

The article is organized as follows. In Section 2, we outline the classical non-equilibrium thermodynamics of a dilute suspension of Brownian particles. In Section 3 this formalism is extended to incorporate the effect of fluctuations using our extended local equilibrium approach. We derive an entropy balance equation from which the stochastic counterpart of entropy production can be deduced and discuss in some detail simple, yet representative cases such as homogeneous solutions and the overdamping limit. In the light of these developments we revisit in Section 4 the single particle case. In Section 5, we establish a connection between the most relevant thermodynamic quantities at the collective and single particle levels of description, along with a comparison between our results and those of previous studies. The main conclusions and possible future extensions of the present work are discussed in Section 6.

\section{Non-Equilibrium Thermodynamics of Brownian Motion}

In this section, we summarize the classical non-equilibrium thermodynamics of a dilute binary mixture of heavy (Brownian) particles embedded in a solvent of light particles, in the presence of a conservative external force.

\subsection{Conservation of Mass}

The law of mass conservation for such a system reads:

$$
\frac{d \rho_{t}}{d t}=-\rho_{t} \nabla \cdot \mathbf{v}_{t}
$$

where $\rho_{t}$ is the total density of the fluid and $\mathbf{v}_{t}$ is the center of mass velocity defined as

$$
\mathbf{v}_{t}=\left(\frac{\rho_{S}}{\rho_{t}}\right) \mathbf{v}_{S}+\left(\frac{\rho}{\rho_{t}}\right) \mathbf{v}
$$

Here $\rho$ and $\mathbf{v}$ are the mass density and the velocity of the Brownian particles and the subscript $S$ refers to quantities pertaining to the light particles. We will consider isothermal $(d T / d t=0)$ and incompressible $\left(d \rho_{t} / d t=0\right)$ fluids with no bulk motion $\left(\mathbf{v}_{t}=0\right)$. Under these assumptions, the balance equation for the partial densities take the simplified form:

$$
\begin{aligned}
& \frac{\partial \rho_{S}}{\partial t}=-\nabla \cdot \mathbf{J}_{\mathbf{S}} \\
& \frac{\partial \rho}{\partial t}=-\nabla \cdot \mathbf{J}
\end{aligned}
$$

where $\mathbf{J}_{S}=\rho_{S} \mathbf{v}_{S}$ and $\mathbf{J}=\rho \mathbf{v}$ are the diffusive fluxes of the solvent and the Brownian particles, respectively. The latter equation can also be written in terms of the mass fraction of Brownian particles $c=\rho / \rho_{t}$,

$$
\rho_{t} \frac{\partial c}{\partial t}=-\nabla \cdot \mathbf{J}
$$

and similarly for the mass fraction of the solvent. 


\subsection{Equation of Motion}

The evolution equation for the velocity of the Brownian particles is given by the sum of the forces acting on it:

$$
\begin{aligned}
\frac{\partial \mathbf{v}}{\partial t} & =\mathbf{F}-\frac{1}{\rho} \nabla \cdot \mathbf{P}-\beta \mathbf{v} \\
& =\mathbf{F}-\nabla \mu-\beta \mathbf{v} .
\end{aligned}
$$

In the above equation, $\mathbf{F}$ stands for the external force per unit mass, the pressure tensor $\mathbf{P}$ is limited to its hydrostatic part and the $\beta \mathbf{v}$ term, in which $\beta$ is a collective friction coefficient, accounts for the dissipative stress exerted on the Brownian particles by the solvent. The final expression involving the gradient of chemical potential per unit mass

$$
\mu=\mu^{0}+\frac{k_{B} T}{m} \ln \rho,
$$

where $m$ is the mass of a single particle, is established by using the relation $\mathbf{P}=p \mathbf{I}$ where $\mathbf{I}$ is the identity matrix, together with the equation of state for the osmotic pressure in a dilute solution $\left(p=\rho k_{B} T / m\right)$.

\subsection{Conservation of Energy and the First Law}

For systems subjected to time-independent potentials, the local form of conservation of total energy per unit mass $e$ reads

$$
\rho_{t} \frac{\partial e}{\partial t}=-\nabla \cdot \mathbf{J}_{e}
$$

In this equation, $\mathbf{J}_{e}$ is the total flux of energy which reads (remember that $\mathbf{v}_{t}=0$ )

$$
\mathbf{J}_{e}=\psi \mathbf{J}+\mathbf{J}_{q},
$$

where $\psi$ is the external potential from which the force acting on the Brownian particles derives and $\mathbf{J}_{q}$ is the heat flow. In the presence of time-dependent potentials, an additional contribution must be included:

$$
\rho_{t} \frac{\partial e}{\partial t}=-\nabla \cdot\left(\psi \mathbf{J}+\mathbf{J}_{q}\right)+\rho \frac{\partial \psi}{\partial t} .
$$

A balance equation can be obtained on this basis for the local internal energy per unit mass, $u$, by subtracting the potential and kinetic energies of the constituents from the total energy (see [3], Equation (II.32)):

$$
u=e-c \psi-\frac{1}{2} c_{S} \mathbf{v}_{S}^{2}-\frac{1}{2} c \mathbf{v}^{2} .
$$

A short comment is in order here concerning our definition of the internal energy. Traditionally, $u$ is obtained by subtracting from $e$ the barycentric kinetic energy $\frac{1}{2} \rho_{t} \mathbf{v}_{t}^{2}$ rather than the total kinetic energy. The definition we adopt here is in a sense more natural given the privileged role played by the Brownian particles. As it will be shown below it leads to some interesting inertia-related effects. The difference between the two definitions is also discussed in [3] (Chapter III). 
Using (11) in combination with (10) and (5), we arrive at the following expression of the first law of thermodynamics

$$
\begin{aligned}
\rho_{t} \frac{\partial u}{\partial t} & =\rho_{t} \frac{\partial e}{\partial t}-\rho_{t} \psi \frac{\partial c}{\partial t}-\rho_{t} c \frac{\partial \psi}{\partial t}-\rho_{t} \frac{1}{2} \frac{\partial}{\partial t}\left(c_{S} \mathbf{v}_{S}^{2}+c \mathbf{v}^{2}\right) \\
& =-\nabla \cdot \mathbf{J}_{q}+\mathbf{J} \cdot \mathbf{F}-\frac{1}{2} \frac{\partial}{\partial t}\left(\rho_{S} \mathbf{v}_{S}^{2}+\rho \mathbf{v}^{2}\right)
\end{aligned}
$$

where the incompressibility condition has again been used. We observe that internal energy can be exchanged in the form of heat, or produced by the degradation of potential or kinetic energy. For dilute binary solutions containing a single type of Brownian particle as considered here, the velocity of the light particles making up the fluid is $\approx \mathbf{v}_{t}$ since the density $\rho$ is much smaller than that of the solvent. We can thus further reduce (12) to

$$
\rho_{t} \frac{\partial u}{\partial t} \approx-\nabla \cdot \mathbf{J}_{q}+\rho \mathbf{v} \cdot \mathbf{F}-\frac{1}{2} \frac{\partial}{\partial t} \rho \mathbf{v}^{2} .
$$

Notice that friction is accounted for through the presence of the time derivative of $\mathbf{v}$ (see Equation (6)).

\subsection{The Second Law}

To express the second law in a local form we start from Gibbs' equation,

$$
T d s=d u+p d v_{t}-\mu_{S}^{*} d c_{S}-\mu^{*} d c
$$

in which $v_{t}=\rho_{t}^{-1}$ is the specific volume and the $\mu^{*}$ s are the chemical potentials related to the internal energy through the Euler theorem (see [3], Equation (III.34)),

$$
u=T s-p v_{t}+c_{S} \mu_{S}^{*}+c \mu^{*} .
$$

Taking into account the incompressibility condition and the fact that $\mathbf{v}_{S} \approx \mathbf{v}_{t}$, the balance equation for the entropy per unit mass $s$ reads:

$$
\rho_{t} \frac{\partial s}{\partial t}=-\nabla \cdot\left(\frac{\mathbf{J}_{q}-\mu \mathbf{J}}{T}\right)-\frac{\rho}{T} \mathbf{v} \cdot\left(\nabla \mu-\mathbf{F}+\frac{\partial \mathbf{v}}{\partial t}\right),
$$

where $\mu=\mu^{*}+\mathbf{v}^{2} / 2$ is the traditional (non-inertial) expression for the chemical potential. The first term in the right hand side of Equation (16) represents the entropy flux and the second one is the entropy production,

$$
\sigma=-\frac{\rho}{T} \mathbf{v} \cdot\left(\nabla \mu-\mathbf{F}+\frac{\partial \mathbf{v}}{\partial t}\right)
$$

Notice that the chemical potential of the solvent does not appear in the first of the terms between parentheses since we consider dilute systems, for which $\mu_{S}$ can be approximated by a constant. We note that the entropy production (17) has the traditional form consisting of the product of a flux (here proportional to $\mathbf{v}$ ) and the corresponding thermodynamic force, which includes a contribution due to inertia through the acceleration of the Brownian particles. The entropy production can be seen as the superposition of three contributions:

- The dissipation due to the thermodynamic force associated to diffusion, $-T^{-1} \rho \mathbf{v} \cdot \nabla \mu$.

- The dissipation due to the conversion of potential energy into internal energy, $T^{-1} \rho \mathbf{v} \cdot \mathbf{F}$.

- The production of entropy related to the transformation of kinetic energy into internal energy, $-T^{-1} \rho \mathbf{v} \cdot \partial \mathbf{v} / \partial t$. 
Moreover, combining (17) and (6), we find

$$
\sigma=\frac{\beta \rho \mathbf{v}^{2}}{T} .
$$

Note that the positivity of entropy production imposes $\beta \geq 0$.

It should be emphasized that in order to evaluate $\mathbf{v}$ and thus entropy production explicitly, the evolution equation for density (4) is needed. In this context, it is instructive to consider in more detail the overdamping limit $d \mathbf{v} / d t=0$. One then has from (6)

$$
\mathbf{v}=\frac{\mathbf{F}-\nabla \mu}{\beta}=\frac{\mathbf{F}}{\beta}-\frac{k_{B} T}{\beta m \rho} \nabla \rho
$$

where we have used Equation (7). In such case, the mass balance Equation (4) becomes

$$
\frac{\partial \rho}{\partial t}=D \nabla^{2} \rho-\frac{1}{\beta} \nabla \cdot \rho \mathbf{F},
$$

in which one finds the usual Fickian diffusion term with $D=k_{B} T / \beta m$ as well as a transport term due to the external force $\mathbf{F}$. The entropy production takes a rather simple form in this limit,

$$
\sigma=\mathbf{J}_{d} \cdot[\nabla \mu-\mathbf{F}] .
$$

where the effective diffusive flux is given by $\mathbf{J}_{d}=-D \nabla \rho-\rho \mathbf{F} / \beta$.

\section{Stochastic Thermodynamics: Extended Local Equilibrium Approach}

We now extend the above analysis to include the effect of fluctuations, placing ourselves in the framework of the extended local equilibrium hypothesis [12,13]. The evolution equations for the internal energy or the entropy take the same form as in (13) and (16), with the exception that the state variables satisfy now stochastic instead of deterministic evolution laws. In particular, Equation (6) is to be replaced by

$$
\frac{\partial \mathbf{v}}{\partial t}=\mathbf{F}-\nabla \mu-\beta \mathbf{v}+\mathbf{f}(\mathbf{x}, t)
$$

where $\mathbf{f}(\mathbf{x}, t)$ is a random force modeled as a spatiotemporal Gaussian white noise with $\overline{f_{i}(\mathbf{x}, t)}=0$ and $\overline{f_{i}(\mathbf{x}, t) f_{j}\left(\mathbf{x}^{\prime}, t^{\prime}\right)}=Q^{2} \delta\left(\mathbf{x}-\mathbf{x}^{\prime}\right) \delta\left(t-t^{\prime}\right) \delta_{i, j}^{K r}, Q^{2}$ being the intensity of noise and the bar denoting average over realizations of the noise. Equation (22) is reminiscent of fluctuating hydrodynamics [20], in which the hydrodynamic equations are augmented by stochastic forcings satisfying fluctuation-dissipation type relations.

Combining (22) and (14) one can derive an extended form of Equation (16) from which a stochastic entropy production can be identified. The latter is now the sum of two contributions:

$$
\sigma=-\frac{\rho}{T} \mathbf{v} \cdot\left(\nabla \mu-\mathbf{F}+\frac{\partial \mathbf{v}}{\partial t}\right)=\frac{\rho \mathbf{v}}{T} \cdot[\beta \mathbf{v}-\mathbf{f}(\mathbf{x}, t)]=\frac{\beta \rho \mathbf{v}^{2}}{T}-\frac{\rho \mathbf{v}}{T} \cdot \mathbf{f}(\mathbf{x}, t) .
$$

The first term in the last equality, to which we will refer as the systematic part, has exactly the same structure as the deterministic entropy production (18) and is consequently always non-negative, despite the fact that $\mathbf{v}$ is now a stochastic variable. The second term (the fluctuating part) contains a contribution in which the random force appears explicitly and is thus not necessarily positive at all 
times and positions. On the other hand, as it will be shown below the mean of the total stochastic entropy production at steady state,

$$
\begin{aligned}
\bar{\sigma} & =\frac{1}{T}\left[\beta \overline{\rho \mathbf{v}^{2}}-\overline{\rho \mathbf{v} \cdot \mathbf{f}(\mathbf{x}, t)}\right] \\
& =\frac{1}{T}[\overline{\rho \mathbf{v} \cdot \mathbf{F}}-\overline{\rho \mathbf{v} \cdot \nabla \mu}],
\end{aligned}
$$

is always non-negative as required by the second law of thermodynamics and equal to zero at equilibrium where $\mathbf{F}=\nabla \mu=0$. Notice that in the absence of the random force our formulation reduces naturally to classical irreversible thermodynamics. This connection is lacking in most of the current formulations of stochastic thermodynamics, in which entropy production is defined in terms of probabilities of particle trajectories and those obtained by time reversal.

In order to evaluate explicitly the statistical properties of the fluctuating entropy production from expression (23), an evolution equation for both $\mathbf{v}$ and $\rho$ are required. The evolution of $\mathbf{v}$ is given by the Langevin Equation (22) while $\rho$ can be obtained via (4). In the most general case, one is thus confronted to a set of coupled stochastic differential equations, the solution of which is not trivial (note that Equation (4) contains no fluctuating force so that the $\mathbf{v}-\rho$ system is degenerate). A considerable simplification arises when the density can be considered constant in space and time. The Langevin equation for the velocity simplifies then to

$$
\frac{\partial \mathbf{v}}{\partial t}=\mathbf{F}-\beta \mathbf{v}+\mathbf{f}(\mathbf{x}, t)
$$

and $Q^{2}=2 \beta k_{B} T / m$ by virtue of the fluctuation-dissipation theorem. The entropy produced locally over a time interval $\tau$ can be evaluated explicitly since it is entirely determined by $\mathbf{v}$ and by the external force:

$$
T \Delta_{i} s=\rho \int_{0}^{\tau}\left[\mathbf{F}-\frac{\partial \mathbf{v}}{\partial t}\right] \cdot \mathbf{v} d t
$$

Due to the absence of a diffusive force, there are only two contributions left in the entropy production. Below, we evaluate $\Delta_{i} s$ in some representative cases where we limit ourselves to 1-dimensional systems for clarity of presentation.

\subsection{No External Force}

This case corresponds to a system in the absence of constraints, which is thus bound to relax towards its equilibrium state. The entropy produced over any time interval $\tau$ is then related to the variation of the local kinetic energy density:

$$
T \Delta_{i} s=-\rho \int_{0}^{\tau} \frac{\partial \mathrm{v}}{\partial t} \mathrm{v} d t=-\frac{\rho}{2} \int_{0}^{\tau} \frac{\partial \mathrm{v}^{2}}{\partial t} d t=\frac{\rho}{2}\left(\mathrm{v}_{0}^{2}-\mathrm{v}_{\tau}^{2}\right)
$$

Since $\mathrm{v}_{0}$ and $\mathrm{v}_{t}$ are stochastic processes, $\Delta_{i} s$ can take positive as well as negative values. A positive entropy production corresponds to situations where the Brownian particles release part of their kinetic energy to the fluid, which will then start to heat. This is the behavior expected from a classical deterministic approach to the thermodynamics of this system. However, the stochastic entropy production can also be negative when the Brownian particles extract energy from their surrounding to accelerate, which in some sense could be seen as an "anti-thermodynamic" behavior. A question of interest is to quantify the relative probability to observe the two aforementioned situations.

We notice to this end that Equation (25) with $\mathrm{F}=0$ defines an Ornstein-Uhlenbeck process for the velocity $\mathrm{v}$, whose stationary distribution is a zero-mean Gaussian with a variance equal to $k_{B} T / m$. 
Inasmuch as the initial condition is sampled over the stationary probability distribution, (26) can thus be rewritten as

$$
\Delta_{i} s=\frac{k_{B} n}{2}\left[\frac{\mathrm{v}_{0}^{2}}{\operatorname{Var}(\mathrm{v})}-\frac{\mathrm{v}_{\tau}^{2}}{\operatorname{Var}(\mathrm{v})}\right],
$$

where $n=N / V$ is the number density. $\Delta_{i} s$ is in this case (up to a multiplicative factor) the difference between two $\chi^{2}$ random variables whose mean and variance are equal to 1 and 2 , respectively. For sampling times $\tau \gg 0, \mathrm{v}_{0}$ and $\mathrm{v}_{\tau}$ can be considered as independent variables since the correlation of Ornstein-Uhlenbeck processes decreases exponentially fast. The moment-generating function of

$$
\mathrm{Z}=\left[\frac{\mathrm{v}_{0}^{2}}{\operatorname{Var}(\mathrm{v})}-\frac{\mathrm{v}_{\tau}^{2}}{\operatorname{Var}(\mathrm{v})}\right]
$$

can then be factorized as [21]

$$
M_{Z}(r) \equiv \overline{e^{Z r}}=(1-2 r)^{-\frac{1}{2}}(1+2 r)^{-\frac{1}{2}} \quad(2 r>1) .
$$

This result can be rewritten as

$$
M_{Z}(r)=\left(\frac{\frac{1}{4}}{\frac{1}{4}-r^{2}}\right)^{\frac{1}{2}}
$$

which is the moment-generating function of a generalized Laplace (or variance- $\gamma$ ) distribution [22]

$$
M_{Z}(r)=e^{\mu r}\left(\frac{\alpha^{2}-\beta^{2}}{\alpha^{2}-(\beta+r)^{2}}\right)^{\lambda},
$$

for the special case $\mu=\beta=0, \alpha=1 / 2$ and $\lambda=1 / 2$. The probability distribution corresponding to (29) is a modified Bessel function of the second kind,

$$
P(Z)=K_{0}\left(\frac{|Z|}{2}\right)
$$

Re-introducing the original variables, we finally obtain the probability density of entropy production

$$
P\left(\Delta_{i} s\right)=\frac{1}{\pi n k_{B}} K_{0}\left(\left|\frac{\Delta_{i} s}{n k_{B}}\right|\right)
$$

The above analysis can easily be extended to the case of 2- or 3-dimensional systems. Notice that Equation (32) refers to the probability density of the entropy produced by a collection of Brownian particles embedded in a fluid. Interestingly, similar expressions were found for the probability distribution of the heat exchanged by a single Brownian particle [23-25].

The distribution (32) is symmetric with respect to its mean $\overline{\Delta_{i} s}=0$, as shown in Figure 1, which is consistent with the fact that the system is fluctuating around equilibrium. Moreover, the moments generated by this distribution depend only on the density of the system and on universal constants and are in this sense generic. In particular, the standard deviation of the entropy produced is given by

$$
\sqrt{\operatorname{Var}\left(\Delta_{i} s\right)}=n k_{B}
$$

and is thus a multiple of $k_{B}$. A similar conclusion was reached for the equilibrium state of homogeneous reactive systems [13]. Most of the probability mass is centered around 0 while large deviations are penalized exponentially since for large values of entropy production, the probability scales as 
$\exp \left(-\left|\Delta_{i} s\right|\right) / \sqrt{\left|\Delta_{i} s\right|}$. The symmetry of the distribution implies that at equilibrium, the system spends an equal amount of time in the thermodynamic and the "anti-thermodynamic" regimes. This symmetry should break down under non-equilibrium conditions, as it will be seen in the next subsection.

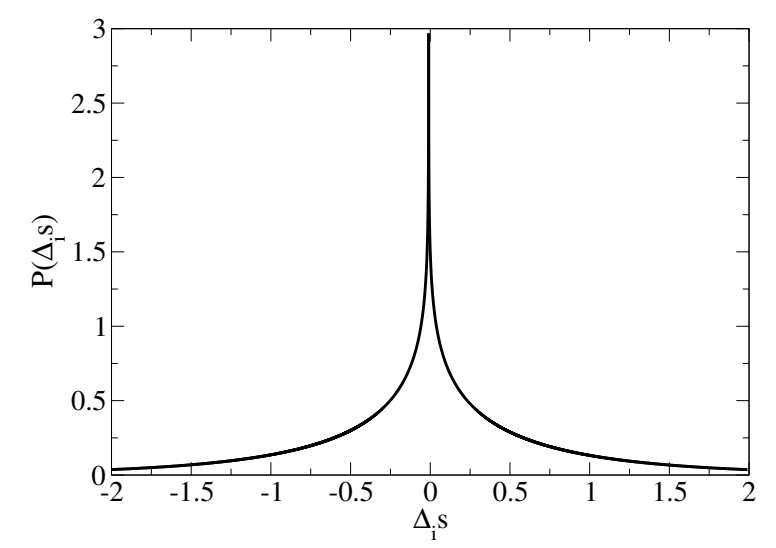

Figure 1. Modified Bessel function of the second kind corresponding to the distribution (32) where $\Delta_{i} s$ is expressed in units of $n k_{B}$.

\subsection{Overdamping Limit}

We next consider the overdamping limit, keeping still the assumption of homogeneous systems. Setting $d \mathrm{v} / d t=0$ in Equation (25), we are led to:

$$
\beta \frac{d x}{d t}=\mathrm{F}+\mathrm{f}(\mathrm{x}, t)
$$

A common choice for $\mathrm{F}$ is to consider that it corresponds to the force exerted by an harmonic trap moving at a given constant velocity $\mathrm{v}^{*}$, for which $\psi=\frac{k}{2}\left(\mathrm{x}-\mathrm{v}^{*} t\right)^{2}$ where $k$ is a constant so that

$$
T \Delta_{i} s=\rho \int_{0}^{\tau} \mathrm{F} \frac{d \mathrm{x}}{d t} d t=-\rho \int_{0}^{\tau} k\left(\mathrm{x}-\mathrm{v}^{*} t\right) \frac{d \mathrm{x}}{d t} d t
$$

It is convenient to introduce a new variable $\mathrm{z}=\mathrm{x}-\mathrm{v}^{*} t[16,17]$, whose evolution equation is

$$
\beta \frac{d z}{d t}=-k z-\beta v^{*}+f(x, t) .
$$

This equation defines again an Ornstein-Uhlenbeck process, whose stationary mean and variance are $-\beta \mathrm{v}^{*} / k$ and $2 k_{B} T / \beta m$, respectively. The entropy production reads

$$
\begin{aligned}
T \Delta_{i} s & =-\rho \int_{0}^{\tau} k \mathrm{z}\left(\frac{d \mathrm{z}}{d t}+\mathrm{v}^{*}\right) d t \\
& =\frac{\rho k}{2}\left(\mathrm{z}_{0}^{2}-\mathrm{z}_{\tau}^{2}\right)-\rho k \mathrm{v}^{*} \int_{0}^{\tau} \mathrm{z} d t .
\end{aligned}
$$

We obtain a superposition of a process whose probability density is a modified Bessel function as above, and of the integral of an Ornstein-Uhlenbeck process. We expect this last contribution to dominate for long times. Moreover, since the covariance of this process decreases exponentially with time, the integral in Equation (36) converges to a generalized Wiener process so that the distribution of entropy production is expected to be Gaussian for long times. This transition can be confirmed numerically for the harmonic force considered here (see Figure 2) and is actually observed even for parameter values beyond the range of validity of the overdamping approximation. 


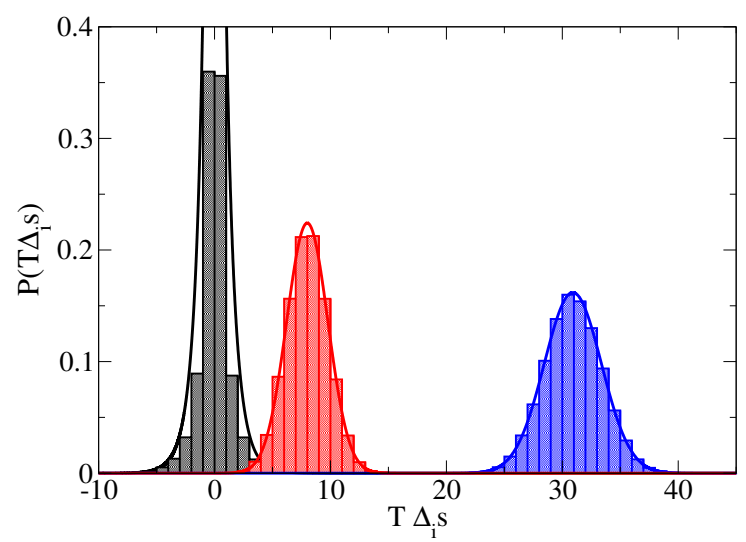

Figure 2. Snapshots of the distribution of the entropy production $T \Delta_{i} s$ obtained from 50,000 realizations of a numerical integration of Equation (22) and substitution in (23). We used a spatially-resolved Euler-Maruyama scheme with a spatial step $d x=0.1$ and a temporal step $d t=10^{-6}$ in a system of linear size $L=200$. As discussed earlier, the integral is to be taken in the sense of Stratonovich [12]. The parameter values are: $\rho=\beta=1, k_{B} T=0.01, k=2$ and $\mathrm{v}^{*}=0.1$. The value of the entropy production has been taken in $x=50$ at $t=1 \times 10^{-4}$ (black histogram), $t=5 \times 10^{-2}$ (red histogram) and $t=0.1$ (blue histogram). The black dashed curve is the modified Bessel function (32), while the red and blue ones are Gaussian fittings. For clarity of presentation, the values of entropy production have been divided by $k_{b} \tau$.

Due to the Gaussian character of its probability distribution, $\Delta_{i} s$ can take positive as well as negative values, like in the equilibrium case considered in the previous section. A negative entropy production corresponds here to a displacement $\mathrm{z}$ that would not be aligned with the external constraint. However, contrary to the situation encountered in Section 3.1, the symmetry of the probability mass in the positive and negative regions is lost. The stationary probability distribution of the time-averaged entropy production,

$$
\sigma_{\tau}=\frac{\Delta_{i} S}{\tau}
$$

reads

$$
P\left(\sigma_{\tau}\right)=\sqrt{\frac{\tau}{2 \pi \Delta_{\sigma}}} \exp \left[-\tau \frac{\left(\sigma_{\tau}-\overline{\sigma_{\tau}}\right)^{2}}{2 \Delta_{\sigma}}\right]
$$

with $\overline{\sigma_{\tau}}=\rho \beta\left(\mathrm{v}^{*}\right)^{2} / T$ and $\Delta_{\sigma}=2 k_{B} \rho^{2}\left(\mathrm{v}^{*}\right)^{2} \beta /(m T)$, since we consider the long-time limit. The probability to observe negative entropy production is thus given by

$$
P\left(\sigma_{\tau}<0\right)=\frac{1}{2}-\frac{1}{2} \operatorname{erf}\left[\sqrt{\frac{\tau}{2 \Delta_{\sigma}}} \overline{\sigma_{\tau}}\right]
$$

which in the limit of large $\tau$ reduces to

$$
P\left(\sigma_{\tau}<0\right) \approx \sqrt{\frac{\Delta_{\sigma}}{2 \pi \tau{\overline{\sigma_{\tau}}}^{2}}} \exp \left(-\frac{\tau}{2 \Delta_{\sigma}}{\overline{\sigma_{\tau}}}^{2}\right) .
$$

We notice that the probability to have $\sigma_{\tau}<0$ decreases rapidly with the sampling time $\tau$ and with the distance from equilibrium, as expressed by $\overline{\sigma_{\tau}}$. 


\section{Single Particle View}

As mentioned in the introduction, most of the studies on stochastic thermodynamics of Brownian motion are limited to a single particle, for which thermodynamic quantities such as entropy production are often defined ad hoc. On the other hand, in Section 3 we derived an expression for the fluctuating entropy production of a collection of Brownian particles (a binary solution), a system that is much closer to the type of systems considered in classical irreversible thermodynamics. In this section, we take advantage of the insights afforded by this formulation to revisit the case of a single Brownian particle. We first recall some of the prominent stochastic thermodynamic properties as they arise at this level of description.

Consider a single Brownian particle placed in a fluid. The position and velocity of this particle will be denoted by $r$ and $v$, respectively and the Langevin equation describing its motion is

$$
\frac{d v}{d t}=\boldsymbol{F}-\gamma \boldsymbol{v}+\boldsymbol{\xi}(t) .
$$

Here $\boldsymbol{F}$ stands, as before, for an external force per unit mass, $\gamma$ is the single-particle viscous friction coefficient and $\xi(t)$ is a Gaussian white noise such that $\overline{\xi_{i}(t)}=0$ and $\overline{\xi_{i}(t) \xi_{j}\left(t^{\prime}\right)}=2 \gamma k_{B} T m^{-1} \delta(t-$ $\left.t^{\prime}\right) \delta_{i, j}^{K r}$. The last two terms in Equation (41) represent a fluctuating dissipative force $\boldsymbol{F}_{\text {diss }}$ acting on the single Brownian particle. This system has been used extensively in the literature to advance a thermodynamic interpretation of quantities of interest in stochastic thermodynamics $[6,11,14-17,26]$. In this section, we focus on quantities connected with those analyzed in the binary solution case presented in Section 3.

Entropy at the level of a single particle is a rather ambiguous concept, but a quantity of clear thermodynamic significance that can be evaluated unambiguously is work. The work done by the particle on its environment is given by

$$
\begin{aligned}
W & =-m \int_{0}^{\tau}\left[\boldsymbol{F}+\boldsymbol{F}_{d i s s}\right] \cdot \boldsymbol{v} d t \\
& =-m \int_{0}^{\tau} \frac{d \boldsymbol{v}}{d t} \cdot \boldsymbol{v} d t \\
& =\frac{m}{2}\left(\boldsymbol{v}_{0}^{2}-\boldsymbol{v}_{\tau}^{2}\right) \\
& =-\Delta E_{k}
\end{aligned}
$$

where $E_{k}$ stands for the kinetic energy of the particle. This relation is a stochastic version of the work-energy theorem.

Expression (42) can be decomposed into a part related to the external systematic force and a part due to the fluctuating, dissipative one in the form $W=W_{\text {ext }}+W_{\text {diss }}$. Since $\boldsymbol{F}$ derives from an external potential $\psi=\psi(r, t)$ where $r$ is the position of the Brownian particle, one may write the first part as

$$
\begin{aligned}
W_{\text {ext }} & =-m \int_{0}^{\tau} \boldsymbol{F} \cdot \boldsymbol{v} d t \\
& =m \int_{0}^{\tau} \frac{\partial \psi}{\partial r} \cdot \frac{d \boldsymbol{r}}{d t} d t \\
& =m \int_{0}^{\tau}\left[\frac{d \psi}{d t}-\frac{\partial \psi}{\partial t}\right] d t \\
& =m \Delta\left(\psi_{\tau}-\psi_{0}\right)-\int_{0}^{\tau} \frac{\partial(m \psi)}{\partial t} d t \\
& =\Delta E_{p}-\int_{0}^{\tau} \frac{\partial E_{p}}{\partial t} d t
\end{aligned}
$$


in which $E_{p}=m \psi$ stands for the potential energy of the particle. For time-independent potentials, Equation (43) reduces to the difference of potential energy between the final and initial states of a single particle.

The dissipative part reads

$$
\begin{aligned}
W_{\text {diss }} & =-m \int_{0}^{\tau} \boldsymbol{F}_{\text {diss }} \cdot \boldsymbol{v} d t \\
& =m \int_{0}^{\tau}[\gamma \boldsymbol{v}-\boldsymbol{\xi}(t)] \cdot \boldsymbol{v} d t
\end{aligned}
$$

To give a clear mechanical meaning to Equation (44) we observe that the power related to the mechanical work exerted by the fluid on the Brownian particle is given by the surface integral of the product of the stress tensor $\boldsymbol{P}$ and the velocity prevailing at the boundary of the particle, $\boldsymbol{v}_{\Sigma}$ [27]:

$$
\frac{d W_{m e c}}{d t}=-\int_{\Sigma} \boldsymbol{P} \cdot \boldsymbol{v}_{\Sigma} \cdot d \boldsymbol{\Sigma}=-\boldsymbol{v} \cdot \int_{\boldsymbol{\Sigma}} \boldsymbol{P} \cdot d \boldsymbol{\Sigma}
$$

where the particle has been assimilated to a rigid sphere, so that $v_{\Sigma}=v$ is the same at any point on the surface. The stress tensor takes into account the short-ranged interactions between the molecules forming the surface of the Brownian particle and those of the surrounding fluid. In general, this tensor will have an intricate form. However, for the simple equation of motion (41) adopted here for the particle as a whole the integral of the stress tensor can be identified by noting that it reduces to the dissipative force,

$$
-\int_{\boldsymbol{\Sigma}} \boldsymbol{P} \cdot d \boldsymbol{\Sigma}=m[-\gamma \boldsymbol{v}+\boldsymbol{\xi}(t)]
$$

We conclude that the mechanical power is given by

$$
\frac{d W_{m e c}}{d t}=m \boldsymbol{v}_{i}\left[-\gamma \boldsymbol{v}_{i}+\boldsymbol{\zeta}_{i}(t)\right]=-\frac{d W_{\text {diss }}}{d t} .
$$

At the single particle level, $W_{\text {diss }}$ can then be interpreted as (minus) the mechanical work due to the forces acting between the system and its close environment. We also notice that Equation (44) closely resembles that of the integral of the entropy production studied in Section 3 (see Equation (23)). We will discuss this point further in Section 5. For now, we will consider a few representative cases where the different contributions to the work can be evaluated explicitly.

\subsection{Time-Independent Potential}

We first consider the case where the applied potential does not depend explicitly on time, i.e., $\partial E_{p} / \partial t=0$. The different contributions to $W$ now have a simple connection with changes in the energy. The total work still corresponds to the change of kinetic energy, $W=-\Delta E_{k}$, while the external and dissipative contributions are related, respectively, to the variation of potential and of mechanical energy:

$$
\begin{aligned}
W_{\text {ext }} & =\Delta E_{p} \\
W_{\text {diss }} & =W-W_{\text {ext }}=-\Delta E_{k}-\Delta E_{p} .
\end{aligned}
$$

We note in particular that all three contributions are "state functions", in the sense that their value only depends on the initial and final positions and velocities.

As an illustration we consider the case of a 1-dimensional motion of a particle under a constant force, $E_{p}(r)=-m F r$. The Langevin Equation (41) describes now an Ornstein-Uhlenbeck process for $v$, which possesses an invariant probability distribution in the form of a Gaussian centered on 
$\bar{v}_{s t}=F / \gamma$. The probability distribution of the total work $W$ can thus be calculated explicitly using Equation (42):

$$
P_{W}(W)=\int_{0}^{\infty} d v_{0}^{2} \int_{0}^{\infty} d v_{\tau}^{2} P\left(v_{0}^{2}\right) P\left(v_{\tau}^{2}\right) \delta\left(W+\frac{m v_{\tau}^{2}}{2}-\frac{m v_{0}^{2}}{2}\right)
$$

where $\delta$ stands for a Dirac delta function. Sampling initial velocities with the invariant distribution,

$$
P(v, s t)=\sqrt{\frac{m}{2 \pi k_{B} T}} \exp \left[-\frac{k_{B} T}{2 m}\left(v-\frac{F}{\gamma}\right)^{2}\right],
$$

we find in a way similar to what was done in Section 3.1 that the distribution of the total work is a modified Bessel function of the second kind,

$$
P_{W}(W)=\frac{1}{\pi k_{B} T} K_{0}\left(\left|\frac{W}{k_{B} T}\right|\right) .
$$

This is confirmed by numerical simulations summarized in Figure 3. We notice that the total work can be either positive or negative, with equal probability in view of the symmetry of the modified Bessel function, irrespective of the system being at equilibrium or under non-equilibrium constraints.

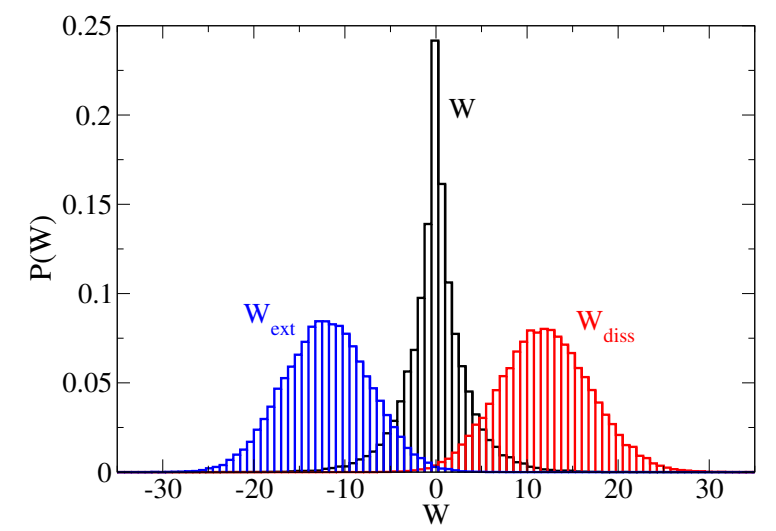

Figure 3. Distribution of the total work $W$, the dissipated work $W_{\text {diss }}$ and the external work $W_{\text {ext }}$ obtained from 50,000 realizations of a numerical integration of Equation (41) followed by substitution in Equations (42)-(43), respectively. We used an Euler-Maruyama scheme with a temporal step $d t=10^{-6}$ for a total time $\tau=12$. The parameter values are $\gamma=k_{B} T=F=1$. Note that to ensure that initial conditions are sampled over the stationary distribution, a relaxation period of 100 time units has been included before statistics were taken.

Interestingly, the two contributions to the total work will typically follow different statistics. The systematic part,

$$
W_{\text {ext }}=\Delta E_{p}=-m F r_{\tau}
$$

corresponds to the integral of an Ornstein-Uhlenbeck process and can thus be seen as a generalized Wiener process in the limit of long times (see again Section 3.1). The probability distribution of $W_{\text {ext }}$ will thus rapidly relax towards a Gaussian distribution, whatever the choice of initial conditions (see Figure 3). $W_{\text {ext }}$ is most often negative, but its probability distribution presents a tail in the 
positive region so that the probability mass to observe a positive value follows a law similar to (40). The dissipated work,

$$
W_{\text {diss }}=W-W_{\text {ext }}=\frac{m v_{0}^{2}}{2}-\frac{m v_{\tau}^{2}}{2}+m F r_{\tau}
$$

is the superposition of a variable whose distribution follows a modified Bessel function (for an initial sampling on the invariant distribution) and of a Wiener process. For short times, the part in $r_{\tau}$ should be small so that the Bessel variable will dictate the statistical properties of $W_{\text {diss }}$, due essentially to changes in the velocity of the heavy particle and thus to inertial effects. On the other hand, the contribution in $r_{\tau}$ should dominate for long times since $r$ can increase without bounds. In such case, the probability distribution will be a Gaussian centered on a moving average $\overline{W_{\text {diss }}}=F^{2} \tau / \gamma$, as illustrated in Figure 3 . We can expect the probability distribution of $W_{\text {diss }}$ to smoothly transform from one form to the other. For Gaussian distributions the dissipated work is positive on average but still has a non-zero probability to be negative, again with a law of the type (40). The interpretation of such events is similar to the multiparticle case: they correspond to situations where the Brownian particle extracts energy from its surrounding and subsequently accelerates. Such events become rare in the long-time limit as the mean of the Gaussian distribution drifts away from zero. In the absence of external force, however, the distribution of $W_{\text {diss }}$ will retain the form of a modified Bessel function for all times, meaning that positive and negative dissipations are equiprobable.

\subsection{Overdamping Limit}

Setting $d v / d t=0$ in Equation (41), we obtain

$$
\gamma \frac{d r}{d t}=\boldsymbol{F}+\xi(t) .
$$

We first note that since the acceleration is zero, the total work vanishes on the grounds of Equation (42), $W=0$, so that the work due to external force and the dissipative work cancel each other exactly at all times:

$$
W_{\text {diss }}=-W_{\text {ext }}
$$

Several typical subcases can be considered. In the case of a constant force in 1 dimension, $W_{\text {ext }}$ and $W_{\text {diss }}$ both follow a Gaussian distribution in the long-time limit since (cf. Equations (49) and (50))

$$
W_{\text {diss }}=-W_{\text {ext }}=m F r_{\tau}
$$

where $r_{\tau}$ is an Ornstein-Uhlenbeck process. Comparing (53) to the solution of the full case (50), we note that the inertial contribution to $W_{\text {diss }}$ has disappeared, as expected. Another typical case corresponds to a moving harmonic trap, for which $\psi=\frac{k}{2}\left(r-v^{*} t\right)$ where $v^{*}$ is a constant trap velocity. This situation is analogous to the case described in Section 3.2. Consequently, we can again introduce a rescaled position $z=r-v^{*} t$ and the dissipated work reads

$$
W_{\text {diss }}=-W_{\text {ext }}=\frac{m k}{2}\left(z_{0}^{2}-z_{\tau}^{2}\right)+m k v^{*} \int_{0}^{\tau} z d t .
$$

We obtain the sum of a term distributed according to a modified Bessel function and another one corresponding to a generalized Wiener process, much like in the binary solution case analyzed in Section 3, see Equation (36). 


\section{Discussion}

In this section, we comment on the connection between the different expressions of the entropy production obtained in the case of a binary solution of Brownian particles and those of the dissipated work for the single Brownian particle.

As Equations (23) and (44) clearly show, the expression for entropy production in the binary solution case and the work-related quantities appearing in the single particle problem bear strong similarities. To connect them more closely, we first consider the case of a 1-dimensional system with a constant density of Brownian particles. The collective velocity $\mathrm{v}$ used in Sections 2 and 3 is then nothing but the sum the velocities of individual Brownian particles, $\mathrm{v}=\sum_{i=1}^{N} v_{i}$, each of which follows a Langevin equation of the type

$$
\frac{d v_{i}}{d t}=F_{i}-\gamma v_{i}+\xi_{i}(t)
$$

with $\overline{\xi_{i}(t)}=0$ and $\overline{\xi_{i}(t) \xi_{j}\left(t^{\prime}\right)}=2 \gamma k_{B} T m^{-1} \delta\left(t-t^{\prime}\right) \delta_{i, j}^{K r}$. In view of Equations (25) and (55), we are led to set

$$
\begin{aligned}
\mathrm{F} & =\sum_{i=1}^{N} F_{i} \\
\beta & =N \gamma \\
f(x, t) & =\sum_{i=1}^{N} \xi_{i}(t) .
\end{aligned}
$$

The global external force is thus the combination of all the forces acting on the individual particles. A similar conclusion holds for the random force $f(x, t)$ and for the global friction coefficient.

We can make use of these relations to rewrite the equation for the entropy production in (23),

$$
\begin{aligned}
\sigma_{N} & =\frac{\rho \mathrm{v}}{T}[\beta \mathrm{v}-f(x, t)] \\
& =\frac{\rho}{T}\left(\sum_{i} v_{i}\right)\left[\sum_{j}\left(\gamma v_{j}-\xi_{j}(t)\right)\right] \\
& =\frac{\rho}{T} \sum_{i, j}\left[\gamma v_{i} v_{j}-\xi_{i}(t) v_{j}\right] .
\end{aligned}
$$

In the limit of a single Brownian particle, this reduces formally to

$$
\sigma_{1}=\frac{\rho}{T}\left[\gamma v_{i}^{2}-\xi_{i}(t) v_{i}\right]
$$

the corresponding dissipated power being

$$
\Delta_{i} S=V \Delta_{i} S=\frac{m}{T} \int_{0}^{\tau}\left[\gamma v_{i}-\xi_{i}(t)\right] v_{i} d t=\frac{W_{\text {diss }}}{T} .
$$

This equation shows that the work dissipated by a single particle can be interpreted in terms of entropy production. More precisely, the single particle entropy production is proportional to the work due to the non-conservative force associated with the interaction between the Brownian particle and the surrounding fluid.

This conclusion is in line with previous thermodynamic interpretations of the single-particle quantities. The external $\left(W_{\text {ext }}\right)$ and dissipative $\left(W_{\text {diss }}\right)$ contributions to the total work exerted by a Brownian particle on the surrounding fluid have been given various meanings in the literature. It was originally proposed to consider that $W_{\text {diss }}$ (44) is a form of particle-level heat [14]. On the other hand, $W_{\text {ext }}$ has also been identified by other authors $[15-17,28]$ with a single-particle equivalent of 
heat in the overdamping limit. As we have shown earlier, these two approaches are in fact equivalent (up to a difference of sign) since in the overdamping limit, $W_{\text {diss }}+W_{\text {ext }}=0$. They are also consistent with our conclusions, provided that one assumes that the particle-level entropy is at a steady state, so that the heat released and the entropy production have identical absolute values.

It should be emphasized that in the approach we developed here, a clearcut connection between entropy production and dissipated work can only be established when there is a single particle in the fluid. In the case of many homogeneously distributed particles the collective entropy production (59) contains coupling terms of the type $v_{i} v_{j}$ and the simple relation between $\Delta_{i} S$ and $W_{\text {diss }}$ is lost, both at the single-trajectory level and on average. Moreover, in the presence of heterogeneities a term proportional to the gradient of chemical potential appears in the Langevin equation for the global velocity (Equation (22)) and thus also in entropy production. These contributions cannot be reproduced with a Langevin equation of the type (55). The dissipated work by itself thus accounts for only a part of the total entropy production.

\section{Conclusions}

In this work the stochastic approach to irreversible thermodynamics recently developed by the present authors was applied to Brownian motion. Work, kinetic and internal energy, heat, entropy and entropy production were analyzed for a dilute binary solution of Brownian particles as well as for a single Brownian particle embedded in a fluid of light particles. Special emphasis was placed on the derivation of the probability distribution of entropy production in the binary solution case and of the probability distributions of the different types of work (external, dissipative and total) in the single particle case, under both equilibrium and nonequilibrium conditions. If the system is fluctuating around equilibrium the distributions of the entropy production and of the total work are symmetric functions centered on zero and the system can be found with equal probability in regions where these quantities have positive or negative values. On the other hand in the presence of nonequilibrium constraints the entropy production and the dissipative work are Gaussian-like distributions possessing a positive mean value, a property that can be regarded as the expression of the second law of thermodynamics. Owing to the presence of fluctuations they still display a tail in the region of negative values, reflecting situations where with some probability the fluxes present may not be aligned to the corresponding forces. The probability of such events is, however, exponentially decreasing with time and with the distance from equilibrium. These properties shed new light on the role of nonequilibrium as a source of time symmetry-breaking.

By systematically comparing the collective and single Brownian particle properties our analysis clarifies a number of issues raised in the literature such as interpreting dissipated work at the single particle level as an equivalent of entropy production, or external work as an equivalent of heat. It also highlights the role of spatial inhomogeneities as reflected by the presence of a term involving the gradient of chemical potential in the expression of entropy production in the binary solution case, which is by necessity absent in a single particle-based approach.

Our approach was based on an extended local equilibrium Ansatz stipulating that in the presence of fluctuations entropy remains a state function of the state variables, which now obey stochastic differential equations extending the familiar evolution equations of the mean-field description. It would be interesting to compare systematically this approach with the one based on a statistical definition of entropy in terms of probability distributions of the state variables, such as the Gibbs-Shannon form used in $[11,18,19]$. In this approach, entropy production is defined in terms of the probability fluxes appearing in the Fokker-Planck equation corresponding to the Langevin dynamics (55). This expression of entropy production misses contributions that we identified with our approach, more specifically terms related to the local density or to its gradient. The reason is that the Gibbs-Shannon definition of entropy used corresponds to an averaging performed over both velocities and positions, in which contributions due to gradients of composition are erased. One way out could be to use instead a local entropy density obtained by averaging only over velocities. A similar approach was used in [3] 
to obtain an expression for the entropy production in binary mixtures in the deterministic limit. Extending this formulation would allow one to account for effects arising from the spatial coupling of Brownian particles and to clarify further the relation between the binary solution and the single particle descriptions.

A more fundamental approach exploring further the connection between stochastic thermmodynamics and fluctuating hydrodynamics, in which the phenomenological friction term and the associated random force used throughout this work are expressed in terms of the dissipative stress tensor and the associated random momentum flux [20,27], would also be desirable. This would in turn necessitate to go beyond the classic Landau-Lifshitz approach in which entropy is a non-fluctuating quantity defined in terms of ensemble probabilities and to introduce one way or another a generalized, fluctuating entropy.

Acknowledgments: This work was supported, in part, by the European Space Agency and the Belgian Federal Science Policy Office.

Author Contributions: Both authors contributed equally to this work. Both authors have read and approved the final manuscript.

Conflicts of Interest: The authors declare no conflict of interest.

\section{References}

1. Glansdorff, P.; Prigogine, I. Thermodynamic Theory of Structure, Stability and Fluctuations; John Wiley \& Sons: New York, NY, USA, 1971.

2. Nicolis, G.; Prigogine, I. Self-Organization in Nonequilibrium Systems; John Wiley \& Sons: New York, NY, USA, 1977.

3. De Groot, S.R.; Mazur, P. Non-Equilibrium Thermodynamics; Dover Publications: Mineola, NY, USA, 2011.

4. Luo, J.-L.; Van den Broeck, C.; Nicolis, G. Stability Criteria and Fluctuations around Nonequilibrium States. Z. Phys. B Condens. Matter 1984, 56, 165-170.

5. Mou, C.Y.; Luo, J.-L.; Nicolis, G. Stochastic Thermodynamics of Nonequilibrium Steady States in Chemical Reaction systems. J. Chem. Phys. 1986, 84, 7011-7017.

6. Seifert, U. Entropy Production along a Stochastic Trajectory and an Integral Fluctuation Theorem. Phys. Rev. Lett. 2005, 95, 040602.

7. Ge, H. Extended forms of the second law for general time-dependent stochastic processes. Phys. Rev. E 2009, $80,021137$.

8. Santillán, M.; Qian, H. Irreversible thermodynamics in multiscale stochastic dynamical systems. Phys. Rev. E 2011, 83, 041130.

9. Van den Broeck, C. Stochastic thermodynamics: A brief introduction. Phys. Complex Colloids 2013, 184, 155-193.

10. Van den Broeck, C.; Esposito, M. Ensemble and trajectory thermodynamics: A brief introduction. Physica A 2015, 418, 6-16.

11. Seifert, U. Stochastic thermodynamics, fluctuation theorems and molecular machines. Rep. Prog. Phys. 2012, $75,126001$.

12. De Decker, Y.; García Cantú Ros, A.; Nicolis, G. Extended local equilibrium approach to stochastic thermodynamics. Eur. Phys. J. Spec. Top. 2015, 224, 947-968.

13. De Decker, Y.; Derivaux, J.-F.; Nicolis, G. Stochastic thermodynamics of reactive systems: An extended local equilibrium approach. Phys. Rev. E 2016, 93, 042127.

14. Sekimoto, K. Stochastic Energetics; Lecture Notes in Physics; Springer: Berlin, Germany, 2010; Volume 799.

15. Van Zon, R.; Cohen, E.G.D. Stationary and transient work-fluctuation theorems for a dragged Brownian particle. Phys. Rev. E 2003, 67, 046102.

16. Andrieux, D.; Gaspard, P.; Ciliberto, S.; Garnier, N.; Joubau, S.; Petrosyan, A. Entropy Production and Time Asymmetry in Nonequilibrium Fluctuations. Phys. Rev. Lett. 2007, 98, 150601.

17. Andrieux, D.; Gaspard, P.; Ciliberto, S.; Garnier, N.; Joubau, S.; Petrosyan, A. Thermodynamic time asymmetry in non-equilibrium fluctuations. J. Stat. Mech. 2008, 2008, P01002. 
18. Tomé, T.; de Oliveira, M.J. Entropy production in irreversible systems described by a Fokker-Planck equation. Phys. Rev. E 2010, 82, 021120.

19. Tomé, T.; de Oliveira, M.J. Stochastic approach to equilibrium and nonequilibrium thermodynamics. Phys. Rev. E 2015, 91, 042140.

20. Landau, L.D.; Lifshitz, E.M. Fluid Mechanics; Pergamon Press: Oxford, UK, 1987.

21. Abramowitz, M.; Stegun, I.A. Handbook of Mathematical Functions; Dover Publications Inc.: Mineola, NY, USA, 1972.

22. Kotz, S.; Kozubowski, T.; Podgórski, K. The Laplace Distribution and Generalizations; Springer Science + Business Media: New York, NY, USA, 2001.

23. Imparato, A.; Peliti, L.; Pesce, G.; Rusciano, G.; Sasso, A. Work and heat probability distribution of an optically driven Brownian particle: Theory and experiments. Phys. Rev. E 2007, 76, 050101.

24. Chatterjee, D.; Cherayil, B.J. Exact path-integral evaluation of the heat distribution function of a trapped Brownian oscillator. Phys. Rev. E 2010, 82, 051104.

25. Crisanti, A.; Sarracino, A.; Zannetti, M. Heat fluctuations of Brownian oscillators in nonstationary processes: Fluctuation theorem and condensation transition. Phys. Rev. E 2017, 95, 052138.

26. Jarzynski, C. Comparison of far-from-equilibrium work relations. C. R. Phys. 2007, 8, 495-506.

27. Bedeaux, D.; Mazur, P. Brownian motion and fluctuating hydrodynamics. Physica 1974, 76, 247-258.

28. Mazonka, O.; Jarzynski, C. Exactly solvable model illustrating far-from-equilibrium predictions. arXiv 1999, arXiv:cond-mat/9912121.

(C) 2017 by the authors. Licensee MDPI, Basel, Switzerland. This article is an open access article distributed under the terms and conditions of the Creative Commons Attribution (CC BY) license (http:/ / creativecommons.org/licenses/by/4.0/). 